\title{
The Use of CLBA Scores in LINC Program Placement Practices in Western Canada
}

\section{Marian Rossiter and Grazyna Pawlikowska-Smith}

Results of a survey of 19 ESL/LINC programs in western Canada regarding the use of CLBA scores for in-class placement purposes are provided. The authors present and discuss factors that contribute to the need, where applicable, for additional placement testing by some programs, and how these factors are in part related to different functions of tests, to varying approaches to scoring (holistic and analytical), and to the format by which the assessment results are communicated. Issues of concern to LINC administrators and recommendations for addressing these concerns are also presented.

Background to the Study

In 1996 the Canadian Language Benchmarks (CLB) was published in response to the "need for a common set of standards to assist in the measurement and description of language skills" (Citizenship and Immigration Canada, 1996, p. 1). The Canadian Language Benchmarks Assessment (CLBA) tool, the second phase of this project, was developed to reflect the levels of skills outlined in the Canadian Language Benchmarks document, and aims at helping to place language learners across the country in instructional programs appropriate to their level of competence in English (Norton Peirce \& Stewart, 1997). Introduced in January 1997, the CLBA, a low-stakes task-based test, measures the lower eight of the 12 levels described by the Canadian Language Benchmarks (Benchmarks 1-8).

The CLBA is administered to adult immigrants to Canada who are eligible for Language Instruction for Newcomers to Canada (LINC, Employment and Immigration Canada, 1991a, 1991b), a program funded by the federal government. The CLBA comprises three components: a Listening/ Speaking assessment, a Reading assessment, and a Writing assessment. The assessments are conducted by certified CLBA assessors, mostly at LINC assessment and referral centers across the country. The average administration time required is 2-2.5 hours. The three resulting scores (Listening/ Speaking, Reading, and Writing) are recorded on the CLBA Client Profile Form as Placement Benchmark Indicators and made available, together with the LINC level Placement Recommendation, to both the applicants and officials in the language programs in which they choose to register.

When a student registers in a language program, a second battery of tests is sometimes administered to the student, thus subjecting many learners in 
the system to two extensive language testing sessions before placement in a particular LINC class. A preliminary inquiry into this situation (Pawlikowska-Walentynowicz, 1997) revealed that many students questioned the need for additional testing, as did some teachers, given the time and resources required. At the same time, some teachers and program coordinators found that CLBA scores did not provide them with the kind of information that they traditionally relied on for placement purposes, hence the perceived need for additional testing beyond the CLBA. The extent to which additional placement tests were used in LINC programs, however, was not clear.

The present study was designed to determine the extent to which CLBA scores are actually used for placement purposes in LINC and to identify the factors that contribute toward the need, where applicable, for further placement testing of LINC students in the programs in which they register.

\section{Method}

\section{Participants}

A stratified sample of LINC programs in Edmonton, Calgary, and Vancouver was selected to participate in this survey. The stratification variables used were city and size of program. The sample was drawn from sampling frames provided by Language Assessment, Referral and Counselling Centre (LARCC); Immigrant, Language and Vocational Assessment-Referral Centre (ILVARC); and Western ESL Services, in Edmonton, Calgary, and Vancouver respectively. The six institutions chosen from each city comprised a total of five large (with more than 200 students each), eight medium (numbering between 100 and 199 students), and six small programs (of 1-99 students), based on February 1998 LINC registration data.

The 19 programs included in the study represented a total of 14 full-time and 16 part-time programs: 12 institutions offered both full-time and parttime classes, two offered full-time classes only, and four offered part-time classes only. One large institution, which used the CLBA for placement in its part-time but not in its full-time program, has been counted as two separate programs. The number of learners represented by these programs totalled 3,223: 874 in Edmonton, 1,069 in Calgary, and 1,280 in Vancouver. The respondents from programs with classes at several locations were asked to restrict their comments to their on-site program so as to ensure that comparisons were made among programs operating according to similar parameters. By including a number of programs of varying sizes, both private and publicly-funded, we hoped to gain a more comprehensive representation of the extent to which CLBA scores were used for placement purposes in western Canada. 


\section{Development and Administration of the Survey}

A questionnaire was developed to elicit information regarding program placement procedures and the extent to which CLBA scores were used for this purpose. It was submitted for review to an expert in applied measurement and evaluation and to an applied linguist in a Teaching English as a Second Language (TESL) MEd program. As a result of this review, modifications were made to the wording and format of several questions. The revised survey consisted of 11 questions and provided opportunity for additional comments for purposes of elaboration and/or clarification. Questions addressed program parameters, curriculum, and number of LINC students registered; the familiarity of survey participants and program staff with the CLBA; the degree of integration of Canadian Language Benchmarks in the program; the extent to which CLBA Placement Recommendations were used for placement purposes and perceived as useful; the characteristics of additional tests that were administered for placement purposes by institutions; discrepancies between official CLBA scores and final placement in the program; and any related issues that respondents wished to raise.

We contacted program directors initially in person or by telephone to solicit their participation. All who were approached kindly agreed to participate in the survey. Interviewers spoke to two representatives at one institution, three at another, and one at each of the remaining 16. Representatives included two teachers delegated to participate because of their extensive experience in the LINC program, three administrators responsible for placement in LINC programs, and 16 directors of LINC programs. They received a letter explaining the study, a consent form ensuring confidentiality, and a questionnaire prior to a telephone interview. These telephone interviews ranged in length from 20-40 minutes and were conducted by the authors during March and April 1998.

\section{Results}

The results of the survey were tabulated, and comments were grouped into general categories according to issues identified and recommendations presented. These are provided below in the order in which the corresponding questions appeared in the survey.

\section{Familiarity with CLBA}

Familiarity with the CLBA was judged on the basis of whether program staff had attended a 2-3-hour orientation session organized by the local assessment center (LARCC, ILVARC, Western ESL Services); staff in all but one program had taken part in one of these sessions. Those who had attended were asked if the orientation session had enhanced the interpretation and usefulness of CLBA scores for placement purposes (see Table 1); five par- 
Table 1

Usefulness of CLBA Orientation Session

\begin{tabular}{lcrcc}
\hline Program size & Yes & Partly & No & n/a \\
\hline Large $(n=5)$ & 0 & 4 & 0 & 1 \\
Medium $(n=8)$ & 1 & 4 & 3 & 0 \\
Small $(n=6)$ & 4 & 1 & 1 & 0 \\
\hline Total $(n=19)$ & 5 & 9 & 4 & 1 \\
\hline
\end{tabular}

ticipants responded affirmatively, four answered negatively, and nine felt that the sessions had been partly successful. Respondents from 10 institutions indicated that although the workshops had promoted understanding of the CLBA and its relation to LINC programs, the overviews presented were too general, too brief, and/or too infrequent to facilitate continuing familiarity. Security issues limited the amount of information that had been shared during these sessions, so many questions remained unanswered. One participant recommended that a demonstration of the administration and scoring of the test would have been useful, and another suggested that a second edition of the CLBA be constructed so that the first edition of the CLBA might become available for ongoing orientation purposes.

\section{Use of CLBA Scores for Placement Purposes}

Frequency of use of the CLBA is reported in Table 2. Respondents from 14 of the 19 programs-two large, seven medium, and five small-reported using CLBA scores for student placement. In two of these programs institutional tests were administered only to students whose initial Placement Recommendation seemed inappropriate, for example, those who demonstrated greater proficiency in the classroom setting than was indicated by their Placement Recommendation.

Students in five other programs-three large, one medium, and one small-were placed into LINC classes on the basis of commercial or in-house tests. The rationale provided for this was, however, extensive. First, two administrators of programs with classes at more than three proficiency levels found that institutional tests provided more discrete information for placement purposes. In addition, interviews of LINC clients conducted on site yielded personal information that was useful for teachers (e.g., age, educational background, previous ESL experience, emotional state, readiness for learning). Speaking tests also gave staff in three programs an opportunity to evaluate grammatical correctness, complexity, fluency of speech, listening comprehension, and auditory acuity. Two respondents reported that general 
Table 2

Use of CLBA Scores Alone for Placement Purposes

\begin{tabular}{lcc}
\hline Program size & Yes & No \\
\hline Large $(n=5)$ & 2 & 3 \\
Medium $(n=8)$ & 7 & 1 \\
Small $(n=6)$ & 5 & 1 \\
\hline Total $(n=19)$ & 14 & 5 \\
\hline
\end{tabular}

vocabulary was assessed by means of interviews and multiple-choice tests, and in three programs writing samples were obtained for placement and diagnosis of literacy skills. In one program the institutional placement test was also administered as an exit test to measure student progress. For all these reasons representatives from the five programs where commercial or in-house tests were administered to all incoming LINC students felt that the time and expense incurred were justified.

\section{Usefulness of CLBA scores}

Of the 14 programs that used CLBA scores alone for placement purposes, respondents from eight considered them "very useful" for placement purposes, and six reported them "somewhat useful" (see Table 3). Scores provided information to teachers on learners' relative strengths and weaknesses among the three skills (oral, reading, writing). One respondent reported that teachers who were required to assign a Benchmark level to students exiting the program also found that the CLBA Client Profile scores were helpful guidelines for reassessment purposes.

Table 3

Usefulness of CLBA Scores

\begin{tabular}{lccc}
\hline Program size & $\begin{array}{c}\text { Very } \\
\text { useful }\end{array}$ & $\begin{array}{c}\text { Somewhat } \\
\text { useful }\end{array}$ & $\begin{array}{c}\text { Not } \\
\text { useful }\end{array}$ \\
\hline Large $(n=5)$ & 2 & 0 & 3 \\
Medium $(n=8)$ & 4 & 3 & 1 \\
Small $(n=6)$ & 2 & 3 & 1 \\
\hline Total $(n=19)$ & 8 & 6 & 5 \\
\hline
\end{tabular}


Table 4

Discrepancy Between CLBA Recommendation and Final Placement

\begin{tabular}{lccccc}
\hline Program size & $0-4 \%$ & $5-10 \%$ & $11-15 \%$ & $35 \%$ & $n / a$ \\
\hline Large $(n=5)$ & 0 & 2 & 0 & 0 & 3 \\
Medium $(n=8)$ & 3 & 4 & 0 & 0 & 1 \\
Small $(n=6)$ & 1 & 2 & 1 & 1 & 1 \\
\hline Total $(n=19)$ & 4 & 8 & 1 & 1 & 5 \\
\hline
\end{tabular}

\section{Discrepancy Between CLBA Recommendation and Final Placement}

Only representatives of the 14 programs that relied on CLBA scores for placement were asked to address this issue. Respondents from four programs indicated that almost no discrepancies occurred, eight estimated that $5-10 \%$ of their students had to be transferred, one estimated $15 \%$, and one estimated $35 \%$ (see Table 4 ). The four programs with the fewest discrepancies were medium programs, and the two with the greatest number of discrepancies were small.

\section{Placement Level}

The placement discrepancies that occurred were reported at all levels. In eight programs the discrepancy occurred at (a) particular level(s): one program reported discrepancies at Pre-LINC and Level 1; one at Level 1; one at Levels 1 and 2; two at Level 2; two at Level 3; and one at Level 3+. Eight respondents voiced the concern that a small minority of students did not perform to the best of their ability on the CLBA because of factors such as culture shock or the desire to qualify for maximum funding. One spoke of the occasional student who entered the program with a Pre-Benchmark designation (minimal literacy skills), but once in class demonstrated the ability to write full paragraphs. Another cited variance between assessors (low interrater reliability) as a possible cause of discrepancy.

\section{Skill Area}

Of the 14 programs that used CLBA Placement Recommendations to place students into programs, respondents of 10 found that there was no particular skill area in which these discrepancies manifested themselves; of the remaining four, two indicated discrepancies in Listening/Speaking, one in Writing, and one in both Reading and Writing. 


\section{Issues of Concern to Respondents}

Participants in the survey were asked if they had additional comments that they would like to make concerning the CLBA. The broad range of proficiency within recommended levels was cited by five interviewees as problematic for teachers. It was suggested that more discrete scoring would be helpful (e.g., 3.1, 3.2, 3.3) in the creation of more homogeneous classes, that the division of the lower levels into LIT 1,2, and 3 would be helpful, and that there should be some way to indicate borderline cases (e.g., where a difference of one point would change the Placement Recommendation). Concern was expressed by one individual that score accuracy and consistency were lacking in the CLBA for a variety of reasons, some admittedly beyond control (e.g., test anxiety, deliberate underperformance, culture shock).

Six respondents reiterated that the separate scores received for Listening/ Speaking, Reading, and Writing were useful. Four respondents expressed a strong desire for division of the Listening/Speaking score to reflect differences between receptive and productive language skills. Five respondents suggested that writing samples be provided to receiving institutions, that grammar skills be tested, and/or that more information, both personal and language-based, be available to LINC program administrators for placement purposes.

There was some variation in the quantity of comments provided by assessment centers about the Client Profile Form, in addition to Placement Benchmark Indicators and Placement Recommendation. Respondents from four institutions in one city stated that they received useful diagnostic information on the CLBA Client Profile (e.g., "weak oral skills"; "student is much higher than score indicates"); this was considered especially useful where there was a wide disparity in skill levels or where students were at either extreme end of the proficiency scale (Preliteracy or LINC 3+). The assessment centers in the other two cities were reported to furnish only administrative comments (e.g., "300 hours at LINC 2"; "withdrew due to pregnancy"), or more often none at all. One representative of a large program voiced the opinion that she would in fact not like to receive "free-hand" comments from assessors who for the most part had no background in ESL. Instead, she recommended a comprehensive checklist (similar to that used in the Canadian Language Benchmarks Literacy Assessment) of specific characteristics of students' communicative performance (e.g., "fluent but very incorrect"; "writes in simple but complete sentences"), as well as a sample of student writing and separate scores for listening comprehension, grammatical accuracy, grammatical complexity, and fluency.

Two program administrators observed that with use of the CLBA, the language competence ceiling has risen, and the top classes are of higher proficiency than before. Students with overall proficiency beyond the LINC 
program, but with one score still in the LINC range (Benchmark 8 or below), now qualify for the federally funded LINC program, whereas those with more homogeneous high proficiency levels must pay for similar programming. One respondent noted that as a result, eligibility for the LINC program has increased, but there is some duplication of services provided by other programs in the same institution.

Four administrators in one city stated that they were expected to assess students as they exited the LINC program but had difficulty doing this without appropriate assessment tools and tasks. One administrator advocated that standardized tests based on the Benchmarks be made available for assessment of progress in programs; another suggested a checklist be developed for this purpose. It should be noted, however, that with the implementation of practices such as these, the Benchmarks would risk becoming a covert curriculum, thus inducing LINC instructors to "teach to the test" for accountability purposes (Brindley, 1998).

In summary, two or more respondents to the survey recommended that the following be provided to officials of LINC programs: (a) more detailed, analytic scores regarding client language proficiency (e.g., grammatical accuracy and fluency indicators); (b) substantive comments on client skills (e.g., "confident"; "self-corrects"; "no past tense"); (c) separate assessment scores for Listening and Speaking on the Client Profile Form; (d) client writing samples for diagnostic instructional purposes; (e) information on the client's educational background, previous ESL experience, length of time in Canada, age, and so forth; and (f) a full sample test, as well as CLBA-type materials and assessment tools for classroom purposes.

\section{Discussion}

One of the major limitations of this study is that it was based on a sample of LINC programs in three major cities in Alberta and British Columbia alone. This sample did, however, include more than half the LINC providers in those centers, which served well over 3,000 students, and we feel that many of the practices described will be of relevance to other LINC programs throughout Canada. We were pleased with the response from the institutional representatives contacted and with the detailed, constructive information that they provided.

The results of the survey indicate that a large number (14 out of 19) of the LINC programs in this sample base placement decisions solely on CLBA scores ( 5 out of 6 small programs, 7 out of 8 medium programs, and 2 out of 5 large programs). It should be noted, however, that the three large institutions that do not use CLBA scores alone provide LINC instruction to 1,080 of 3,223 clients, or about one third of the learners in this sample. Representatives of the five programs that use in-house placement tests report that CLBA scores do not provide enough information (e.g., a measure of gram- 
matical accuracy) to place clients at appropriate levels in their curricula. When an extensive centralized test such as the CLBA does not provide adequate detail regarding an individual for placement purposes, its value for users may be uncertain. Four issues seem to underlie this uncertainty.

\section{Issue \#1. The intended purpose and function of the CLBA:}

Proficiency or placement?

Language tests are used for different primary purposes and, as Brindley (1998) points out, unless all stakeholders share a common understanding of the assessment rationale, confusion could exist regarding the official intended purpose of a test. The introductory booklet to the CLBA (Bergin, da Silva, Peirce, \& Stewart, 1996) states that the purpose of the CLBA is "to help place language learners across the country in instructional programs appropriate for their level of competence in English" (p. 3). It is clear, then, that the intended function of the CLBA is assessing general proficiency; no claims are made to placement within programs.

The function of proficiency assessment in an educational setting is to help administrators and teachers make decisions that are concerned not with in-class placement but rather with student selection; these include the general fit of students and program (e.g., LINC), entrance and exit standards, and the relationship of the program to other educational systems. For these functions a proficiency test like the CLBA, which uses broad ability bands and provides an overall indication of proficiency, is an appropriate instrument to use.

Issue \#2. Appropriateness of criterion-referenced test information for placement decisions in programs

The goal of placement decisions is to group students into homogeneous classes (in terms of defined abilities, learning points to be addressed, and program curriculum), as well as to stream learners according to their learning profiles, needs, and goals to achieve the best match with instructional techniques and practices. In order to do this, a comfortably wide spread of scores to identify differences between individual performances in selected areas of proficiency (together with additional learner profile information) is required. One type of test that generates a wide distribution of scores is a norm-referenced test. Some test experts claim that proficiency or placement decision functions can only be achieved satisfactorily with a norm-referenced rather than a criterion-referenced test. Brown (1996) writes: "I argue that proficiency decisions should be made on the basis of norm-referenced tests because NRTs have all the qualities desirable for such decisions" (p. 10). Bachman (1990) recommends that if a placement test is designed on the basis of a theory of language proficiency, a norming procedure should be used. If, however, the placement test is based on the learning objectives of the syllabus, and well-specified and sequenced objectives are available, the test 
could be criterion-referenced-the criterion would be defined as mastery of the objectives at each level of the program.

Our survey indicates that the CLBA, a criterion-referenced test, seems to achieve reasonable success as a placement instrument in smaller programs that provide instruction at lower levels of basic proficiency. It does not produce a wide distribution of scores, however, so the in-class placement function in many cases cannot be realized. For instance, in large LINC programs where the intake of new students consists of 100 individuals with the same LINC 3 placement recommendation based on the CLBA scores, it is necessary to gather additional information to differentiate between individuals for in-class placement decisions.

\section{Issue \#3. CLBA scoring scheme and reporting format: Limitations for in-class placement}

The traditional criteria used for the assessment of language abilities or their components can be treated analytically or holistically (globally) in the rating scheme of a test. Global and analytic scoring schemes differ in their capability to provide the necessary detail for in-class placement. How the assessment results are reported to the users (the reporting format) is also of paramount importance for in-class placement decisions.

In an analytic scheme the components are listed and assessed separately; impressions regarding effectiveness, fluency, appropriateness, grammatical accuracy, lexical range, coherence, and so forth are each assessed separately on a point scale and aggregated or weighted proportionally to derive a composite score. Thus analytic scoring provides detailed information about various aspects of learners' performance and of their relative strengths and weaknesses. Holistic scoring, on the other hand, is a global assessment of the overall effectiveness of learner performance in which separate assessments of individual components are collapsed into global impression bands.

The CLBA employs global impression bands in the form of benchmark descriptors. For example, the CLBA Listening/Speaking Benchmark 4 descriptor (as it appears on the back of the CLBA Client Profile Form) reads as follows:

Adequate competence in basic oral communication. Can describe the process of obtaining essential goods and services. Needs little support from assessor. Understands instructions. Understands short sentences. Clear evidence of connected discourse. Conveys messages using a variety of short sentences. Has control of basic grammar structures and tenses. Pronunciation difficulties may impede communication. Vocabulary adequate for basic oral communication.

The global impression method of scoring in ESL assessment reflects the limitations of existing models of second language proficiency and its acquisi- 
tion. Band models, including the CLB, cannot account for the multidimensionality of individual performance. Individuals have differing degrees of control over different components of language ability (fluency, accuracy, structures, vocabulary) and display various configurations of ability within one global range, band, or benchmark level of an assessment scale. Weir (1993) argues that

Given that our understanding of the continuum of proficiency is currently limited, we might be better served for the moment by analytical scales, where assessments are made in terms of each criterion separately... It would seem imprudent to rely on band scales which collapse criteria together and assume that students progress equally in all criteria as they move up the band scale. (p. 45)

He reiterates: "the global approach fails in practice because it does not cater for learners whose performance levels vary in terms of different criteria" ( $p$. 164).

The global impression scoring and, therefore, the global reporting of the scores has definite drawbacks in generating in-class placement information, especially if performed by external assessors. Although, as our survey indicates, LINC programs are largely satisfied with the accuracy of the global benchmark/LINC level assessment that the CLBA provides, and although it is mostly the large programs that feel the need for more detailed, microlinguistic assessment profiles of their learners, there is no doubt that all programs would benefit from having relevant data for placement purposes in closer accordance with what is taught in the classroom. Such separate measures, however, are not provided by the CLBA.

\section{Issue \#4. Practicality considerations}

Test practicality, one of the components of test usefulness (Bachman \& Palmer, 1996), is a function of the available and required resources in test development and test use. In our survey, concern was voiced about the cost of additional placement testing for each program, and support was expressed for the exclusive use of a revised CLBA Client Profile for placement purposes, provided that (a) the scores were more detailed and transparent, (b) other relevant learner placement information was included, and (c) the reporting format was meaningful to providers. Hunter, Jones, and Randhawa (1996) note:

research attention might be paid to the means of communicating holistic scores.... Because professionals must use the scores to adjust their instructional practices, and ultimately to calibrate their classroom evaluation practices with the profiles generated by a large-scale assessment program, their perceptions of report usefulness are important.... extended exemplars of student writing are necessary illustrations of holis- 
tic scoring criteria. Assessors often do not include this descriptive documentation in reports.... so that reports are less meaningful to an assessment's audiences. (p. 81)

The score-reporting aspect of assessment, whether holistically or analytically derived, is of paramount importance to the educational system as a whole.

\section{Conclusions and Recommendations}

The CLBA as a proficiency test was designed with a specific purpose in mind: to select students for placement in appropriate programs. In its present form it appears to have only limited usefulness as an in-class placement instrument. It is criterion-referenced and reports broad-band proficiency information; it does not, however, discern small differences between individuals' performances, a necessary requirement for placement decisions in some-especially larger-programs.

Three options exist with regard to program needs for in-class placement information and the issues of practicality and resources raised in the present study:

1. Once learners are placed in appropriate programs (e.g., LINC, ESL literacy, labor market skill training programs), additional tests could continue to be administered where necessary to gather further information for in-class placement purposes. A proficiency test is not the only appropriate instrument in the teaching-testing operation, and a combination of tests is needed to supply particular information at various levels of analysis in some LINC programming situations. As Spolsky (1995) writes:

A helpful analogy to language testing is in the field of pain measurement and assessment.... Rather than expecting some simple mechanical device to translate the complex data of individual language proficiency into a single measure, language testers, too, would benefit from intelligent and responsible "interpretations drawn from patterns evident among the combined measures." (pp. 350-351)

2. Because the cost efficiency of additional testing is an issue, the idea of the dual assessment function (proficiency/placement) for the CLBA is indeed attractive. In order for the CLBA to combine the two functions successfully, however, the test would have to be adapted to yield and to report additional specific analytic and diagnostic information for some of the test tasks. All reported assessment information would need to relate to the criteria that teachers understand and use in placement, instruction, and assessment, and it would have to be communicated in sufficient detail and in a clear, standard format. 
3. With proper confidentiality controls in place, CLBA assessment centers and assessors are in a position to provide ESL programs with essential client placement information collected during needs assessment interviews (e.g., language background, educational background, prior language learning experience, future goals, language use needs, occupational/professional information, and special learning needs) that would assist providers further in placing LINC clients in their programs. This would complement Option A or B practices.

With the establishment of the Center for Canadian Language Benchmarks, future CLBA initiatives are anticipated. Among these, our survey suggests, field practitioners would welcome a further refinement of the CLBA instruments in consultation with program providers in order to obtain more detailed assessment profiles for the placement of learners and to maximize the benefits of the centralized assessment at both system and classroom levels. The CLBA Client Profile, the standard score reporting format, could be expanded to include analytic performance indicators (commonly used and well understood in ESL placement contexts) as well as qualitative information. Training of current CLBA assessors in the use of these profiles could be provided as part of a regular process to ensure continuing assessor interreliability. Additional CLBA orientation materials could also be developed to illustrate more fully the criterion tasks, assessment procedures, and scoring of responses at different levels of proficiency and to facilitate understanding of the CLBA among teachers. In conclusion, the implementation of some of the recommendations put forward in this study could well foster greater reliance on CLBA scores and enhance the overall efficiency of placement procedures in LINC and other ESL programs throughout Canada.

\section{Acknowledgments}

The authors would like to thank all the LINC program representatives who participated in this study. We are grateful to Tracey Derwing, Todd Rogers, and the anonymous TESL Canada Journal reviewers for their comments on earlier versions of this article. We also express our appreciation to Ingrid Hauck and Phil Nagy for making the CLBA technical report available to us.

\section{The Authors}

Marian Rossiter is a doctoral candidate in the Department of Educational Psychology, University of Alberta. Her interests include second-language learning, motivation, research, and testing.

Grazyna Pawlikowska-Smith coordinates CLBA assessment for LINC programs in Edmonton and works as a sessional lecturer in the Department of Educational Psychology at the University of Alberta. Her interests include lexical semantics, proficiency, testing, curriculum, and evaluation. Grazyna wrote the revised Canadian Language Benchmarks working document. 


\section{References}

Bergin, M., da Silva, T., Peirce, B.N., \& Stewart, G. (1996). Introduction to the Canadian Language Benchmarks assessment. Mississauga, ON: Peel Board of Education.

Bachman, L.F. (1990). Fundamental considerations in language testing. Oxford, UK: Oxford University Press.

Bachman, L.F., \& Palmer, A.S. (1996). Language testing in practice. Oxford, UK: Oxford University Press.

Brindley, G. (1998). Outcomes-based assessment and reporting in language learning programs: A review of the issues. Language Testing, 15, 45-85.

Brown, J.D. (1996). Testing in language programs. Upper Saddle River, NJ: Prentice Hall Regents. Employment and Immigration Canada. (1991a). Innovations in training. Hull, QC: Author. Employment and Immigration Canada. (1991b). New immigrant language training policy. Hull, QC: Author.

Hunter, D.M., Jones, R.M., \& Randhawa, B.S. (1996). The use of holistic versus analytic scoring for large-scale assessment of writing. Canadian Journal of Program Evaluation, 11(2), 61-87.

Norton Peirce, B., \& Stewart, G. (1997). The development of the Canadian Language Benchmarks assessment. TESL Canada Journal, 14(2), 17-31.

Pawlikowska-Walentynowicz, G. (1997, October). CLBA learner profiles vis-à-vis other placement scores: A preliminary inquiry. Paper presented at the Alberta Teachers of English as a Second Language (ATESL) Conference, Calgary.

Spolsky, B. (1995). Measured words. Oxford, UK: Oxford University Press.

Weir, C. (1993). Understanding and developing language tests. Hemel Hempstead, UK: Prentice Hall Europe. 